\title{
Entropic magmas, their homology and related invariants of links and graphs
}

\author{
MACIEJ NIEBRZYDOWSKI \\ JÓZEF H PRZYTYCKI
}

\begin{abstract}
We define link and graph invariants from entropic magmas, modeling them on the Kauffman bracket and Tutte polynomial. We define the homology of entropic magmas. We also consider groups that can be assigned to the families of compatible entropic magmas.
\end{abstract}

55N35; 18G60, 57M25

\section{Introduction}

A binary operation satisfying the entropic property $(a * b) *(c * d)=(a * c) *(b * d)$ was probably first considered by Sushkevich in 1937 [12]. Soon after, Murdoch [6] and Toyoda in a series of papers $[13 ; 14 ; 15 ; 16]$ established the main properties of such magmas. In particular, they proved the following result named after them; it is very important in our considerations.

Theorem 1.1 $[6 ; 13]$ If $(X ; *)$ is an entropic quasigroup, then $X$ has an abelian group structure such that $a * b=f(a)+g(b)+c$, where $f, g: X \rightarrow X$ are commuting group automorphisms.

The phrase entropic property that we use was coined in 1949 by Etherington [3]. Other names for this property include: mediality, bicommutativity, alternation, bisymmetry and abelianity. The word entropic refers to inner turning.

In the first part of the paper we show how to use the entropic property to define invariants of links and graphs that are based on the Kauffman bracket and the Tutte polynomial so that the assumption of linearity is not present in their construction. In the second part we introduce homology of entropic magmas and its counterpart for families of entropic magmas connected by a condition $\left(a *_{i} b\right) *_{j}\left(c *_{i} d\right)=\left(a *_{j} c\right) *_{i}\left(b *_{j} d\right)$. 


\section{Tait graphs}

We recall here (see eg [8]) a construction of Tait which gives a bijection between signed plane graphs and link diagrams; a bijection which also sends a Kauffman bracket version of the Tutte polynomial to the Kauffman bracket of a link diagram.

Tait was the first to notice the relation between knots and planar graphs. He colored the regions of a knot diagram alternately white and black (motivated by Listing [5]) and constructed a graph by placing a vertex inside each white region, and then connecting vertices by edges going through the crossing points of the diagram (we place however a vertex in black regions in our considerations, and study a "black" graph instead of a dual "white" graph of Tait; Figure 1).

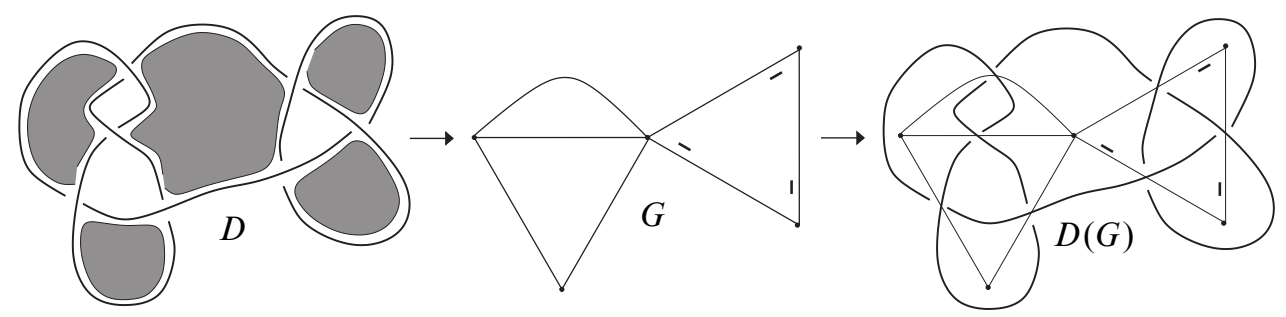

Figure 1: Tait's construction of a graph from a link diagram (connected sum of the figure-eight knot and the left-handed trefoil knot with white infinite region) and back to the signed graph

It is useful to mention the Tait construction going in the opposite direction, from a signed planar graph, $G$, to a link diagram $D(G)$. We replace every edge of a graph by a crossing according to the convention of Figure 2 and connect endpoints along edges as in Figure 1.
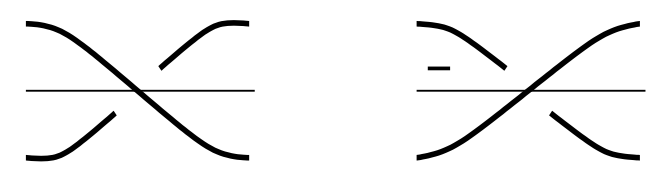

Figure 2: Convention for crossings assigned to signed edges (edges without markers are assumed to be positive)

\section{Entropic magma}

We define link diagram invariants from entropic magmas by modeling them on the Kauffman bracket polynomial (but not assuming linearity). In 1984, entropic magmas 
were used for the first time in knot theory to generalize the Jones and Homflypt link invariants using Conway's skein triple; see the work of Przytycki and Traczyk $[10 ; 11]$ and the survey articles [7; 9]. If we generalize the Kauffman bracket relation, then in order to have link invariants (for framed links) from a magma $(A ; *)$, we need an entropic condition, and an additional condition for the second Reidemeister move. We analyze these below and if initial conditions are given by $\left(a_{1}, \ldots, a_{n}, \ldots\right)$, we call the magma a bracket (or Kauffman bracket) magma. Later we consider signed graphs and play an analogous game with invariants coming from a Tutte magma.

\subsection{Kauffman bracket magma}

We consider link invariants taking values in an entropic magma $(A ; *)$ with a chosen sequence of not necessarily different elements $a_{1}, a_{2}, \ldots, a_{n}, \ldots$, which satisfy the following conditions for any $n \geq 1$. The relations are derived from the second Reidemeister moves (assuming that the crossings are ordered and the two new crossings are the last). We get (i) or (ii) as below:

(i) $\left(a_{n+1} * a_{n}\right) *\left(a_{n+2} * a_{n+1}\right)=\left(a_{n+1} * a_{n+2}\right) *\left(a_{n} * a_{n+1}\right)=a_{n}$

This case is illustrated in Figure 3 for $n=1$.

(ii) $\left(a_{n} * a_{n+1}\right) *\left(a_{n+1} * a_{n}\right)=a_{n+1}$

This case is illustrated in Figure 4 for $n=1$.

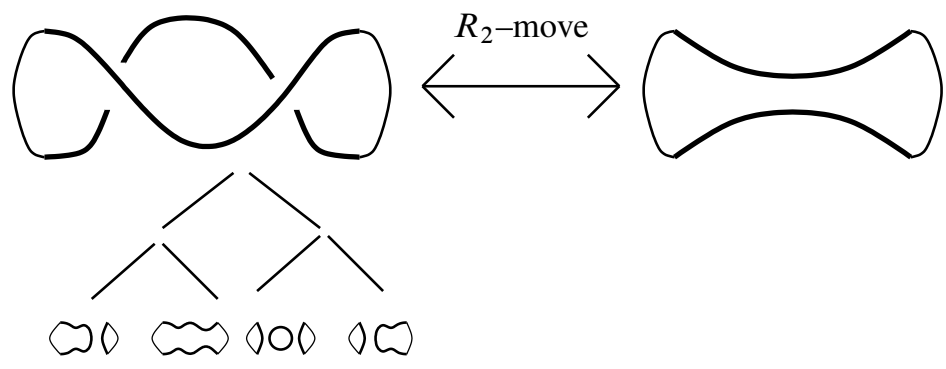

Figure 3: $R_{2}$-move; denominator closure

First, we construct a regular isotopy ${ }^{1}$ link invariant from a Kauffman bracket magma:

\footnotetext{
${ }^{1}$ In regular isotopy we consider unoriented link diagrams modulo the second and the third Reidemeister moves. In an equivalent approach, we can consider unoriented framed links and use the fact that two diagrams on $S^{2}$ representing framed links with blackboard framing are ambient isotopic if and only if they are related by $R_{2}$ and $R_{3}$ moves. Notice that $R_{1}$ changes the framing by \pm 1 .
} 

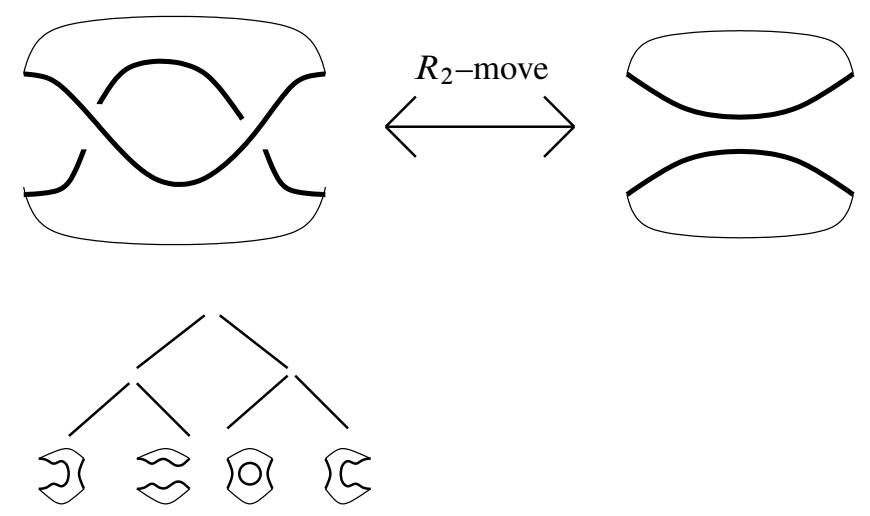

Figure 4: $R_{2}-$ move; numerator closure

Theorem 3.1 Let $\left(A ; *, a_{1}, a_{2}, \ldots\right)$ be a Kauffman bracket magma. Then there is a unique unoriented framed link invariant, say $P:\left\{\right.$ Links $\left.^{f r}\right\} \rightarrow A$, such that $P\left(T_{n}\right)=a_{n}$ and if

$$
\left.L=\searrow, \quad L_{0}=\asymp \text { and } L_{\infty}=\right)(
$$

is a Kauffman bracket skein triple, then

$$
P(L)=P\left(L_{0}\right) * P\left(L_{\infty}\right) .
$$

Proof Our proof follows Kauffman's proof of existence of the Kauffman bracket polynomial but we give it here for completeness. The proof is organized as follows:

(1) We consider any link diagram $D$ representing $L$ and we order its crossings. Then, we compute (in a unique way) the value (in $A$ ) of the diagram, resolving crossings according to their ordering and using initial values at the end (at leaves of the computational tree). We denote this invariant of diagrams with ordered crossings by $P(D)$.

(2) We show that obtained value does not depend on the ordering of crossings. Here, it suffices to check that we can exchange the order of two crossings which were consecutive in the initial ordering, say $v_{1}<v_{2}$. For a diagram $D$ with crossings $v_{1}, v_{2}$, we write $D_{\varepsilon_{1}}^{v_{1}, \varepsilon_{2}}$ for a diagram obtained from $D$ by smoothing $v_{1}$ and $v_{2}$ according to $\varepsilon_{1}, \varepsilon_{2}$, where $\varepsilon_{i}=0$ or $\infty$ decides which Kauffman marker we use for smoothing; of course $D_{\varepsilon_{1}, \varepsilon_{2}}^{v_{1}, v_{2}}=D_{\varepsilon_{2}, \varepsilon_{1}}^{v_{2}, v_{1}}$. We have (in an initial order):

$$
P(D)=P\left(D_{0}^{v_{1}}\right) * P\left(D_{\infty}^{v_{1}}\right)=\left(P\left(D_{0,0}^{v_{1}, v_{2}}\right) * P\left(D_{0, \infty}^{v_{1}, v_{2}}\right)\right) *\left(P\left(D_{\infty, 0}^{v_{1}, v_{2}}\right) * P\left(D_{\infty, \infty}^{v_{1}, v_{2}}\right)\right)
$$


After switching the order of $v_{1}$ and $v_{2}$ we smooth $v_{2}$ first and obtain analogously:

$$
\begin{aligned}
P(D)=P\left(D_{0}^{v_{2}}\right) * P\left(D_{\infty}^{v_{2}}\right) & =\left(P\left(D_{0,0}^{v_{2}, v_{1}}\right) * P\left(D_{0, \infty}^{v_{2}, v_{1}}\right)\right) *\left(P\left(D_{\infty, 0}^{v_{2}, v_{1}}\right) * P\left(D_{\infty, \infty}^{v_{2}, v_{1}}\right)\right) \\
& =\left(P\left(D_{0,0}^{v_{1}, v_{2}}\right) * P\left(D_{\infty, 0}^{v_{1}, v_{2}}\right)\right) *\left(P\left(D_{0, \infty}^{v_{1}, v_{2}}\right) * P\left(D_{\infty, \infty}^{v_{1}, v_{2}}\right)\right)
\end{aligned}
$$

Our invariants coincide on the four diagrams $D_{0,0}^{v_{1}, v_{2}}, D_{0, \infty}^{v_{1}, v_{2}}, D_{\infty, 0}^{v_{1}, v_{2}}$ and $D_{\infty, \infty}^{v_{1}, v_{2}}$. Thus, in order for them to coincide on $D$ it suffices to have an entropic condition. In our case

$$
\begin{aligned}
\left(P\left(D_{0,0}^{v_{1}, v_{2}}\right) * P\left(D_{0, \infty}^{v_{1}, v_{2}}\right)\right) * & \left(P\left(D_{\infty, 0}^{v_{1}, v_{2}}\right) * P\left(D_{\infty, \infty}^{v_{1}, v_{2}}\right)\right) \\
= & \left(P\left(D_{0,0}^{v_{1}, v_{2}}\right) * P\left(D_{\infty, 0}^{v_{1}, v_{2}}\right)\right) *\left(P\left(D_{0, \infty}^{v_{1}, v_{2}}\right) * P\left(D_{\infty, \infty}^{v_{1}, v_{2}}\right)\right)
\end{aligned}
$$

is a special case of the entropic condition.

(3) We analyze the behavior of our diagram invariant under the second Reidemeister move $R_{2}$. Because (by (2)) we can put crossings involved in $R_{2}$ at the very end of the calculations, we conclude that the relations (i) and (ii) of Figure 3 and 4 are necessary and sufficient for invariance.

(4) The invariance under the third Reidemeister move follows from (3), in a similar manner to the case of the Kauffman bracket: we start the resolution from the top crossing; this is illustrated in Figure 5.
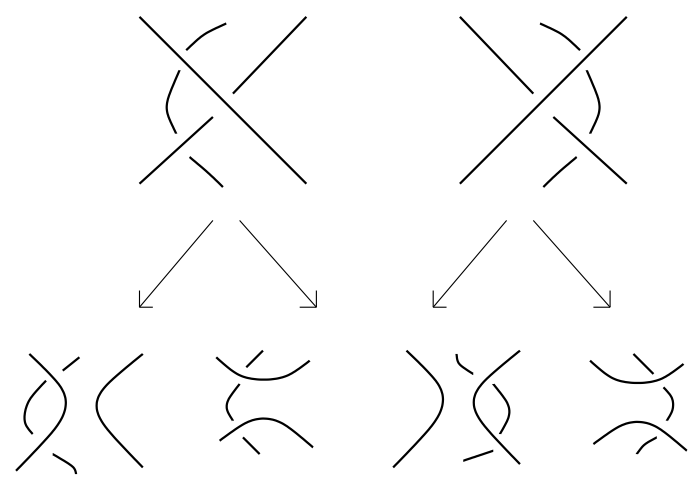

Figure 5: After smoothings of upper crossings, diagrams differ by $R_{2}$ moves only

More precisely, by (2), we can start our calculation from the upper crossing involved in the third Reidemeister move. After the first resolution, the left diagrams differ by two $R_{2}$ moves (so we can use (3)), and the right diagrams are isotopic (Figure 5). 


\subsection{Kauffman bracket magma invariants and first Reidemeister moves}

We discuss in this section how $P(D)$ changes under first Reidemeister moves. We have to consider two types of the first move, a positive $R_{+1}$ in which we add a positive kink, and a negative $R_{-1}$ in which a negative kink is added to a diagram $D$. Equivalently, we measure the effect on $P(L)$ of twisting a framing in a positive direction $\left(L \rightarrow L^{(1)}\right)$ or a negative one $\left(L \rightarrow L^{(-1)}\right)$. Denote by $\tau(D)$ a link diagram $D \sqcup \bigcirc$, that is, we add a trivial component to the diagram $D$. Then we have

$$
\begin{gathered}
P\left(D^{(1)}\right)=P(\diamond)=P(\tau(D)) * P(D), \\
P\left(D^{(-1)}\right)=P(\diamond)=P(D) * P(\tau(D)) .
\end{gathered}
$$

For example, a positive loop (ie the trivial knot with framing $1, T_{1}^{(1)}=\bigcirc$ ) has value $a_{2} * a_{1}$, while $T_{1}^{(-1)}=\bigcirc$ has the value $a_{1} * a_{2}$.

Notice that the equality $P\left(\left(L^{(1)}\right)^{(-1)}\right)=P(L)$ follows from the relation (i) of Section 3.1 even if twisting is performed on different component of $L$. Namely, we can assume that kink crossings are the last crossings of the computational tree, thus we can assume that $L$ is represented by a trivial diagram of $n$ components. Then we have $P\left(L^{(1)}\right)=a_{n+1} * a_{n}, P\left(L^{(-1)}\right)=a_{n} * a_{n+1}$ and

$$
P\left(\left(L^{(1)}\right)^{(-1)}\right)=\left(a_{n+1} * a_{n+2}\right) *\left(a_{n} * a_{n+1}\right) \stackrel{(\mathrm{i})}{=} a_{n}=P(L) .
$$

Let us extend the notation $\tau(L)=L \sqcup \bigcirc$ to a subset of $A$ realized by link invariants. More precisely, let $A^{\mathcal{L}}=\{a \in A \mid a=P(D)$ for some diagram $D\}$. Then on $A^{\mathcal{L}}$ we define $\tau(a)=P(\tau(D))$, where $P(D)=a$. It is not always the case that $\tau$ can be extended from $A^{\mathcal{L}}$ to $A$, but if it does extend, we still denote an extension by $\tau$.

To produce an invariant of unoriented links (diagrams under all Reidemeister moves), we can consider only zero framings on links (that is, the framing given by Seifert surfaces of each component), or we can orient $L$ and consider a framing given by the Seifert surface of the oriented $L$. This can be rephrased using the writhe number of an oriented diagram (the sum of signs of crossings), as was done in the case of the Kauffman bracket (modified to Jones polynomial).

Lemma 3.2 Consider a Kauffman bracket magma $\left(A ; *, a_{1}, a_{2}, \ldots\right)$ that satisfies, for every $n \geq 1$ :

$$
\left(a_{n} * a_{n+1}\right) *\left(a_{n+1} * a_{n+2}\right)=\left(a_{n+2} * a_{n+1}\right) *\left(a_{n+1} * a_{n}\right) .
$$

Then the invariant of links $P(L)$ is preserved by 4-moves. 
Proof We illustrate in Figure 6 how the 4-move changes the Kauffman bracket magma invariant. This involves two variables and can be read as $(a * b) *(b * \tau(b))=$ $(\tau(b) * b) *(b * a)$. But in fact, as we work with diagrams on $R^{2}$, we can smooth all crossings not involved in the move first and then deal exclusively with a clasp, again having two cases, numerator and denominator, as illustrated in Figure 6.
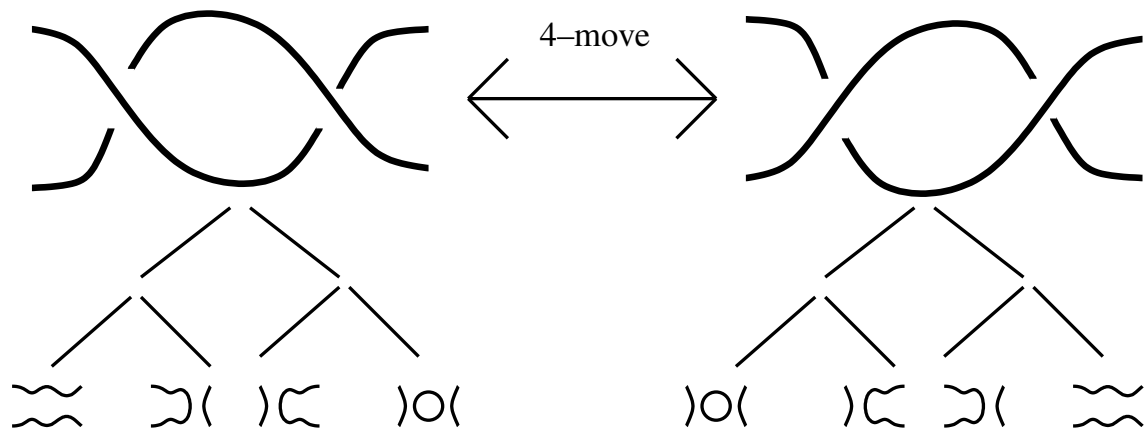

Figure 6: 4-move and resolving computational trees

We also observe that if we work modulo relations giving invariants of 4-moves, then the invariant is preserved by a framing change of multiplicity of four.

\section{Tutte magma for signed graphs}

We are motivated by a bijection between signed plane graphs and link diagrams (on $R^{2}$ or on $S^{2}$, whichever is more convenient), but we define magma invariants for all signed graphs, for any magma $(A ; *)$ with a chosen sequence of elements $a_{1}, a_{2}, \ldots, a_{n}, \ldots$ (not necessarily all different).

If the edges of $G$ are ordered, we define an invariant of a signed graph in Tutte fashion, by giving to a graph with no edges and $n$ vertices (say $T_{n}$ ) the value $a_{n}$ (that is $\left.P\left(T_{n}\right)=a_{n}\right)$, and then:

(i) For a positive edge $e_{+}$which is not a loop:

$$
P(G)=P\left(G / e_{+}\right) * P\left(G-e_{+}\right) .
$$

Here $G-e_{+}$and $G / e_{+}$denote deleting and contracting the edge $e_{+}$, respectively.

(ii) For a negative edge $e_{-}$which is not a loop:

$$
P(G)=P\left(G-e_{-}\right) * P\left(G / e_{-}\right) .
$$


(iii) If $e_{+}$is a positive loop then:

$$
P(G)=P\left(G / / e_{+}\right) * P\left(G-e_{+}\right),
$$

where $G / / e_{+}$is obtained from $G / e_{+}$by adding an isolated vertex.

(iv) If $e_{-}$is a negative loop then:

$$
P(G)=P\left(G-e_{-}\right) * P\left(G / / e_{-}\right),
$$

where $G / / e_{-}$is obtained from $G / e_{-}$by adding an isolated vertex.

When computing the value of $P(G) \in A$, we use the edges of $G$ one by one according to their ordering.

The invariant is well-defined but depends on the ordering of the edges. If we switch the order of two edges, we may have different results: one of the form $(a * b) *(c * d)$, and the second of the form $(a * c) *(b * d)$. Thus, entropic magma may be used to get an invariant of a signed graph. We can try to do better and consider only some entropic relations. For example, if $G$ has 2 edges, the only type of relation which $(A ; *)$ has to satisfy is an entropic relation

$$
\left(a_{n+1} * a_{n}\right) *\left(a_{n+2} * a_{n+1}\right)=\left(a_{n+1} * a_{n+2}\right) *\left(a_{n} * a_{n+1}\right) .
$$

We meet this condition when considering the graph $\stackrel{+}{-\rightarrow},-$ with additional $n-1$ isolated points. Its two computational trees are shown in Figure 7.
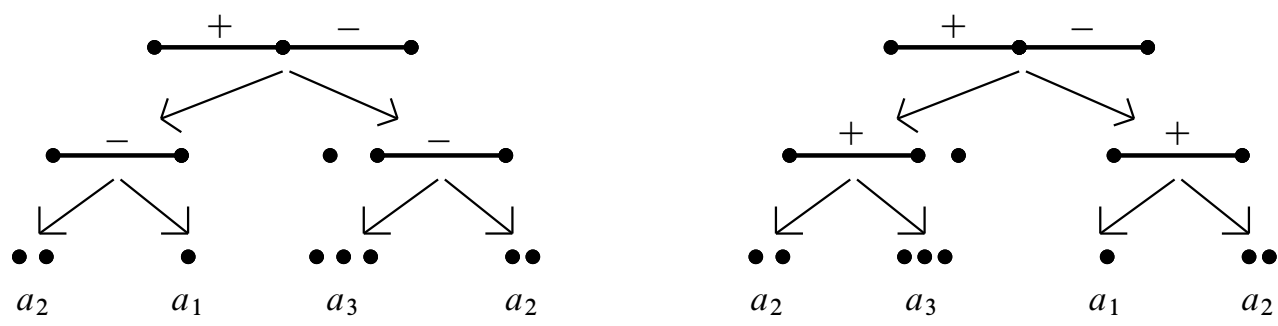

Figure 7: Changing the ordering of edges in a computational tree

When analyzing graphs with 3 edges, we find 4 additional entropic conditions:

By considering the graph

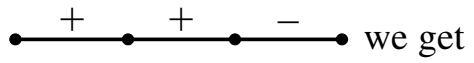

$$
\begin{aligned}
\left(\left(a_{n+2} * a_{n+1}\right) *\right. & \left.\left(a_{n+3} * a_{n+2}\right)\right) *\left(\left(a_{n+1} * a_{n}\right) *\left(a_{n+2} * a_{n+1}\right)\right) \\
& =\left(\left(a_{n+2} * a_{n+1}\right) *\left(a_{n+1} * a_{n}\right)\right) *\left(\left(a_{n+3} * a_{n+2}\right) *\left(a_{n+2} * a_{n+1}\right)\right)
\end{aligned}
$$

and its reverse (by considering $\bullet-, \ldots+,+$, )

$$
\begin{aligned}
\left(\left(a_{n+1} * a_{n+2}\right)\right. & \left.*\left(a_{n} * a_{n+1}\right)\right) *\left(\left(a_{n+2} * a_{n+3}\right) *\left(a_{n+1} * a_{n+2}\right)\right) \\
& =\left(\left(a_{n+1} * a_{n+2}\right) *\left(a_{n+2} * a_{n+3}\right)\right) *\left(\left(a_{n} * a_{n+1}\right) *\left(a_{n+1} * a_{n+2}\right)\right) .
\end{aligned}
$$


From the positive graph $\longrightarrow$ we get an entropic identity

$$
\begin{aligned}
\left(( a _ { n + 1 } * a _ { n } ) * \left(a_{n}\right.\right. & \left.\left.* a_{n+1}\right)\right) *\left(\left(a_{n+2} * a_{n+1}\right) *\left(a_{n+1} * a_{n+2}\right)\right) \\
& =\left(\left(a_{n+1} * a_{n}\right) *\left(a_{n+2} * a_{n+1}\right)\right) *\left(\left(a_{n} * a_{n+1}\right) *\left(a_{n+1} * a_{n+2}\right)\right)
\end{aligned}
$$

and its reverse (by considering the negative graph $\longrightarrow$ )

$$
\begin{aligned}
\left(\left(a_{n+2} * a_{n+1}\right) *\right. & \left.\left(a_{n+1} * a_{n+2}\right)\right) *\left(\left(a_{n+1} * a_{n}\right) *\left(a_{n} * a_{n+1}\right)\right) \\
= & \left(\left(a_{n+2} * a_{n+1}\right) *\left(a_{n+1} * a_{n}\right)\right) *\left(\left(a_{n+1} * a_{n+2}\right) *\left(a_{n} * a_{n+1}\right)\right) .
\end{aligned}
$$

In general, we have to consider all graphs, so there are no easy criteria for setting the conditions. Thus, we define a Tutte magma abstractly as a magma with distinguished elements $a_{1}, a_{2}, \ldots, a_{n}$, such that if we compute the invariants of any fixed signed graph $G$ using different orders of the edges, we always get the same result (we know that we need some, but not all entropic conditions). Formally:

Definition 4.1 Let $\mathcal{A}=\left(A, *, a_{1}, a_{2}, \ldots\right)$ be a magma with a chosen sequence of elements, and $P\left(G^{\text {ord }}\right)$ an associated invariant of graphs with ordered edges. We say that $\mathcal{A}$ is a Tutte magma if $P\left(G^{\text {ord }}\right)$ does not depend on the ordering of edges of $G$, for any signed graph $G$.

Clearly, if $\mathcal{A}$ is an entropic magma, it is also a Tutte magma, but as we demonstrated earlier, not all entropic relations are needed. We could rephrase the definition using partial computational trees for two edges with different orders, but we leave that to the reader. Similarly, we can define partial Tutte magmas (by analogy with partial Conway algebras in $[9 ; 10])$.

Now we describe another invariant of signed graphs, using the function $P$ and congruences on algebras. First, we recall the notion of a congruence on a magma.

Definition 4.2 Let $\mathcal{A}=\left(A, *, a_{1}, a_{2}, \ldots\right)$ be a magma, and let $\theta$ be an equivalence relation on $A$. $\theta$ is a congruence on $\mathcal{A}$ if $b_{1} \theta c_{1}$ and $b_{2} \theta c_{2}$ implies $\left(b_{1} * b_{2}\right) \theta\left(c_{1} * c_{2}\right)$, for $b_{1}, b_{2}, c_{1}, c_{2} \in A$.

Definition 4.3 Given a magma $\mathcal{A}=\left(A, *, a_{1}, a_{2}, \ldots\right)$ and a congruence $\theta$ on $\mathcal{A}$, one can form a quotient magma $\mathcal{A} / \theta$, whose underlying set is the set of equivalence classes of $\theta$, with the binary operation $[a] *[b]=[a * b]$, where $[x]$ denotes the equivalence class of $x \in A$.

Now we are ready to define the invariant. 
Definition 4.4 We begin by fixing a magma $\mathcal{A}=(A, *)$ with a sequence of not necessarily different elements $a_{1}, a_{2}, \ldots$. For any signed graph $G$, let $X_{G} \subseteq A$ denote the set of values of the function $P$ obtained using all possible orderings of the edges of $G$. Let $\theta$ be the smallest congruence on $\mathcal{A}$ generated by the set $X_{G}$ (that is, the smallest congruence containing all the pairs $(a, b)$ for $\left.a, b \in X_{G}\right)$. Form the quotient algebra $\mathcal{A}_{G}=\mathcal{A} / \theta$. Then the function $T$ assigning a quotient algebra $\mathcal{A}_{G}$ to a signed graph $G$ is an invariant of signed graphs.

\subsection{Examples of calculations for line graphs and polygons}

This section contains calculations of $P$ for certain signed plane graphs. We keep in mind the correspondence between such graphs and link diagrams described in Section 2 (especially when using $\tau$ below).

Example 4.5 Let $L_{n}$ be a line graph (that is, a tree with $n+1$ vertices, all except two of degree two), with positive edges. Then we have

$$
P\left(L_{n}\right)=P\left(L_{n-1}\right) * \tau\left(P\left(L_{n-1}\right)\right)
$$

with an initial condition $P\left(L_{0}\right)=a_{1}$. We have for example:

$$
\begin{aligned}
& P\left(L_{1}\right)=P\left(L_{0}\right) * \tau\left(P\left(L_{0}\right)\right)=a_{1} * a_{2}, \\
& P\left(L_{2}\right)=P\left(L_{1}\right) * \tau\left(P\left(L_{1}\right)\right)=\left(a_{1} * a_{2}\right) *\left(a_{2} * a_{3}\right), \\
& P\left(L_{3}\right)=P\left(L_{2}\right) * \tau\left(P\left(L_{2}\right)\right)=\left(\left(a_{1} * a_{2}\right) *\left(a_{2} * a_{3}\right)\right) *\left(\left(a_{2} * a_{3}\right) *\left(a_{3} * a_{4}\right)\right), \\
& P\left(L_{4}\right)=P\left(L_{3}\right) * \tau\left(P\left(L_{3}\right)\right)=\left(\left(\left(a_{1} * a_{2}\right) *\left(a_{2} * a_{3}\right)\right) *\left(\left(a_{2} * a_{3}\right) *\left(a_{3} * a_{4}\right)\right)\right) \\
& *\left(\left(\left(a_{2} * a_{3}\right) *\left(a_{3} * a_{4}\right)\right) *\left(\left(a_{3} * a_{4}\right) *\left(a_{4} * a_{5}\right)\right)\right) \text {. }
\end{aligned}
$$

Example 4.6 Let $C_{n}$ be an $n$-gon (a cycle graph with $n$ edges and $n$ vertices). Then we have

$$
P\left(C_{n}\right)=P\left(C_{n-1}\right) * P\left(L_{n-1}\right)
$$

with an initial condition $P\left(C_{1}\right)=a_{2} * a_{1}$. We have for example:

$$
\begin{aligned}
& P\left(C_{2}\right)=P\left(C_{1}\right) * P\left(L_{1}\right)=\left(a_{2} * a_{1}\right) *\left(a_{1} * a_{2}\right), \\
& P\left(C_{3}\right)=P\left(C_{2}\right) * P\left(L_{2}\right)=\left(\left(a_{2} * a_{1}\right) *\left(a_{1} * a_{2}\right)\right) *\left(\left(a_{1} * a_{2}\right) *\left(a_{2} * a_{3}\right)\right) .
\end{aligned}
$$

$C_{3}$ corresponds to a right handed trefoil knot $\left(\overline{3}_{1}=\varnothing\right)$.

The notation $3_{1}$ is used in the Rolfsen's table of knots for the left-handed trefoil knot 
Example 4.7 Let $C_{n}^{\prime}$ be an $n$-gon with one edge doubled, then we have

$$
P\left(C_{n}^{\prime}\right)=\left(\tau\left(P\left(C_{n-1}\right)\right) * P\left(C_{n-1}\right)\right) * P\left(C_{n}\right) .
$$

For example, if $C_{2}^{\prime}$ is a theta curve and describes the left handed trefoil knot we get

$$
\begin{aligned}
P\left(3_{1}\right)=P\left(C_{2}^{\prime}\right) & =\left(\tau\left(P\left(C_{1}\right)\right) * P\left(C_{1}\right)\right) * P\left(C_{2}\right) \\
& =\left(\left(a_{3} * a_{2}\right) *\left(a_{2} * a_{1}\right)\right) *\left(\left(a_{2} * a_{1}\right) *\left(a_{1} * a_{2}\right)\right) .
\end{aligned}
$$

The graph $C_{3}^{\prime}$ corresponds to the figure eight knot and we have

$$
\begin{aligned}
P\left(4_{1}\right)=P\left(C_{3}^{\prime}\right)=\left(\tau\left(P\left(C_{2}\right)\right) *\right. & \left.P\left(C_{2}\right)\right) * P\left(C_{3}\right) \\
= & \left(\left(\left(a_{3} * a_{2}\right) *\left(a_{2} * a_{3}\right)\right) *\left(\left(a_{2} * a_{1}\right) *\left(a_{1} * a_{2}\right)\right)\right) \\
& *\left(\left(\left(a_{2} * a_{1}\right) *\left(a_{1} * a_{2}\right)\right) *\left(\left(a_{1} * a_{2}\right) *\left(a_{2} * a_{3}\right)\right)\right) .
\end{aligned}
$$

\section{Homology of entropic magmas}

Let $(X ; *)$ be a magma, $A$ any set and $\pi: A \times X \rightarrow X$ the projection to the second factor. Any magma structure on $A \times X$ for which $\pi$ is an epimorphism can be given by a system of functions $\phi_{a_{1}, a_{2}}\left(x_{1}, x_{2}\right): X \times X \rightarrow A$ as

$$
\left(a_{1}, x_{1}\right) *\left(a_{2}, x_{2}\right)=\left(\phi_{a_{1}, a_{2}}\left(x_{1}, x_{2}\right), x_{1} * x_{2}\right) .
$$

The functions $\phi_{a_{1}, a_{2}}\left(x_{1}, x_{2}\right)$ are uniquely defined by the multiplication on $A \times X$. Thus, binary operations on $A \times X$ agreeing with $\pi$ are in bijection with choices of functions $\phi_{a_{1}, a_{2}}$. If we require some special structure on $(X ; *)$ (eg associativity or an entropic condition), we obtain a corresponding property of $\phi_{a_{1}, a_{2}}\left(x_{1}, x_{2}\right)$ which we call a dynamical cocycle for the structure.

(1) Let $(X ; *)$ be a semigroup; in order that an action on $A \times X$ be associative, we need

$$
\begin{aligned}
\left(\left(a_{1}, x_{1}\right) *\left(a_{2}, x_{2}\right)\right) *\left(a_{3}, x_{3}\right) & =\left(\phi_{a_{1}, a_{2}}\left(x_{1}, x_{2}\right), x_{1} * x_{2}\right) *\left(a_{3}, x_{3}\right) \\
& =\left(\phi_{\phi_{a_{1}, a_{2}}}\left(x_{1}, x_{2}\right), a_{3}\left(x_{1} * x_{2}, x_{3}\right),\left(x_{1} * x_{2}\right) * x_{3}\right)
\end{aligned}
$$

to be equal to

$$
\begin{aligned}
\left(a_{1}, x_{1}\right) *\left(\left(a_{2}, x_{2}\right) *\left(a_{3}, x_{3}\right)\right) & =\left(a_{1}, x_{1}\right) *\left(\phi_{a_{2}, a_{3}}\left(x_{2}, x_{3}\right), x_{2} * x_{3}\right) \\
& =\left(\phi_{a_{1}, \phi_{a_{2}, a_{3}}}\left(x_{2}, x_{3}\right)\right. \\
& \left.\left(x_{1}, x_{2} * x_{3}\right), x_{1} *\left(x_{2} * x_{3}\right)\right) .
\end{aligned}
$$

Thus, the dynamical cocycle condition in the associative case has the form

$$
\phi_{\phi_{a_{1}, a_{2}}\left(x_{1}, x_{2}\right), a_{3}}\left(x_{1} * x_{2}, x_{3}\right)=\phi_{a_{1}, \phi_{a_{2}, a_{3}}\left(x_{2}, x_{3}\right)}\left(x_{1}, x_{2} * x_{3}\right) .
$$


(2) We now assume that $(X ; *)$ is an entropic magma, that is $(a * b) *(c * d)=$ $(a * c) *(b * d)$ for any $a, b, c, d \in X$. We look for a condition on the dynamical cocycle so that $A \times X$ is entropic. We need

$$
\begin{aligned}
\left(\left(a_{1}, x_{1}\right) *\left(a_{2}, x_{2}\right)\right) *\left(\left(a_{3}, x_{3}\right) *\left(a_{4}, x_{4}\right)\right) & \\
& =\left(\phi_{a_{1}, a_{2}}\left(x_{1}, x_{2}\right), x_{1} * x_{2}\right) *\left(\phi_{a_{3}, a_{4}}\left(x_{3}, x_{4}\right), x_{3} * x_{4}\right) \\
& =\left(\phi_{\phi_{a_{1}, a_{2}}}\left(x_{1}, x_{2}\right), \phi_{a_{3}, a_{4}}\left(x_{3}, x_{4}\right)\right.
\end{aligned}
$$

to be equal to

$$
\begin{aligned}
\left(\left(a_{1}, x_{1}\right) *\left(a_{3}, x_{3}\right)\right) *\left(\left(a_{2}, x_{2}\right) *\left(a_{4}, x_{4}\right)\right) & \\
& =\left(\phi_{a_{1}, a_{3}}\left(x_{1}, x_{3}\right), x_{1} * x_{3}\right) *\left(\phi_{a_{2}, a_{4}}\left(x_{2}, x_{4}\right), x_{2} * x_{4}\right) \\
& =\left(\phi_{\phi_{a_{1}, a_{3}}}\left(x_{1}, x_{3}\right), \phi_{a_{2}, a_{4}}\left(x_{2}, x_{4}\right)\right.
\end{aligned}
$$

Thus, the dynamical cocycle condition in the entropic case has the form

$$
\begin{aligned}
\phi_{\phi_{a_{1}, a_{2}}\left(x_{1}, x_{2}\right), \phi_{a_{3}, a_{4}}\left(x_{3}, x_{4}\right)}\left(x_{1} * x_{2}, x_{3} * x_{4}\right) \\
=\phi_{\phi_{a_{1}, a_{3}}\left(x_{1}, x_{3}\right), \phi_{a_{2}, a_{4}}\left(x_{2}, x_{4}\right)}\left(x_{1} * x_{3}, x_{2} * x_{4}\right) .
\end{aligned}
$$

Extensions of modules, groups and Lie algebras are described in the classical book by Cartan and Eilenberg [1].

Let $(X ; *)$ be an entropic magma, $A$ an abelian group with a given pair of commuting homomorphisms $t, s: A \rightarrow A$ and a constant $a_{0} \in A$; we consider $(A ; *)$ as an entropic magma with an affine action $a * b=t a+s b+a_{0}$. Then we define a binary operation on $A \times X$ by $\left(a_{1}, x_{1}\right) *\left(a_{2}, x_{2}\right)=\left(a_{1} * a_{2}+f\left(x_{1}, x_{2}\right), x_{1} * x_{2}\right)$. In order for $A \times X$ to be an entropic magma, $\phi_{a_{1}, a_{2}}\left(x_{1}, x_{2}\right)=a_{1} * a_{2}+f\left(x_{1}, x_{2}\right)$ should be an entropic dynamical cocycle. This leads to entropic cocycle condition

$$
\begin{aligned}
& t f\left(x_{1}, x_{2}\right)-t f\left(x_{1}, x_{3}\right)+s f\left(x_{3}, x_{4}\right)-s f\left(x_{2}, x_{4}\right) \\
& +f\left(x_{1} * x_{2}, x_{3} * x_{4}\right)-f\left(x_{1} * x_{3}, x_{2} * x_{4}\right)=0 .
\end{aligned}
$$

The above formula served as a hint in defining entropic homology. In particular, for a unital ring $R, \partial: R X^{4} \rightarrow R X^{2}$ may be given by

$$
\begin{aligned}
\partial\left(x_{1}, x_{2}, x_{3}, x_{4}\right)=t\left(x_{1}, x_{2}\right)-t\left(x_{1}, x_{3}\right)+ & s\left(x_{3}, x_{4}\right)-s\left(x_{2}, x_{4}\right) \\
& +\left(x_{1} * x_{2}, x_{3} * x_{4}\right)-\left(x_{1} * x_{3}, x_{2} * x_{4}\right)
\end{aligned}
$$

and $\partial: R X^{2} \rightarrow R X$ may be given by $\partial\left(x_{1}, x_{2}\right)=t x_{1}-x_{1} * x_{2}+s x_{2}$.

The last map (on the level of cohomology) is derived as follows (we follow the classical case of a group extension by an abelian group; we adjust it to an extension of an entropic magma $(X ; *)$ by an entropic, affine magma $(A ; *))$ : 
We ask when extensions $A \times X \rightarrow X$ given by various $f: X \times X \rightarrow A$ with $\left(a_{1}, x_{1}\right) *\left(a_{2}, x_{2}\right)=\left(a_{1} * a_{2}+f\left(x_{1}, x_{2}\right), x_{1} * x_{2}\right)$ are equivalent in the "fiber preserving" sense. That is, we would like to know whether two extensions $A \times X \rightarrow X$ with the same affine action on $A\left(a * b=t a+s b+a_{0}\right)$ are related by a magma homomorphism $F: A \times X \rightarrow A \times X$, where $F$ is constant on the $X$ factor. We can express $F$ as $F(a, x)=(c(x)+a, x)$, with $c: X \rightarrow A$. "Homomorphism" means, of course, that

$$
F\left(\left(a_{1}, x_{1}\right) *_{1}\left(a_{2}, x_{2}\right)\right)=F\left(a_{1}, x_{1}\right) *_{2} F\left(a_{2}, x_{2}\right) .
$$

Here $*_{1}$ corresponds to $f_{1}$ and $*_{2}$ to $f_{2}$, so we write concretely

$$
\left(a_{1} * a_{2}+f_{1}\left(x_{1}, x_{2}\right)+c\left(x_{1} * x_{2}\right), x_{1} * x_{2}\right)=\left(a_{1}+c\left(x_{1}\right), x_{1}\right) *_{2}\left(a_{2}+c\left(x_{2}\right), x_{2}\right)
$$

and further

$$
\begin{aligned}
\left(a_{1} * a_{2}+f_{1}\left(x_{1}, x_{2}\right)\right. & \left.+c\left(x_{1} * x_{2}\right), x_{1} * x_{2}\right) \\
& =\left(\left(a_{1}+c\left(x_{1}\right)\right) *\left(a_{2}+c\left(x_{2}\right)\right)+f_{2}\left(x_{1}, x_{2}\right), x_{1} * x_{2}\right) \\
& =\left(t\left(a_{1}+c\left(x_{1}\right)\right)+s\left(a_{2}+c\left(x_{2}\right)\right)+a_{0}+f_{2}\left(x_{1}, x_{2}\right), x_{1} * x_{2}\right) \\
& =\left(a_{1} * a_{2}+t c\left(x_{1}\right)+s c\left(x_{2}\right)+f_{2}\left(x_{1}, x_{2}\right), x_{1} * x_{2}\right) .
\end{aligned}
$$

Equivalently,

$$
\left(f_{1}-f_{2}\right)\left(x_{1}, x_{2}\right)=t c\left(x_{1}\right)+s c\left(x_{2}\right)-c\left(x_{1} * x_{2}\right) .
$$

This suggests $(\partial c)\left(x_{1}, x_{2}\right)=t c\left(x_{1}\right)+s c\left(x_{2}\right)-c\left(x_{1} * x_{2}\right)$ and $\left(f_{1}-f_{2}\right)=\partial c$. This further suggests $\partial: R X^{2} \rightarrow R X$ given by

$$
\partial\left(x_{1}, x_{2}\right)=t x_{1}-x_{1} * x_{2}+s x_{2},
$$

as we wrote before. We should mention here that our map $\partial: \operatorname{Hom}\left(R X^{1}, A\right) \rightarrow$ $\operatorname{Hom}\left(R X^{2}, A\right)$ given by

$$
(\partial c)\left(x_{1}, x_{2}\right)=t c\left(x_{1}\right)+s c\left(x_{2}\right)-c\left(x_{1} * x_{2}\right)
$$

does not use any specific properties of the magma $(X ; *)$, thus for $s=1-t$ it gives the coboundary operator for the twisted rack cohomology (see [2]),

$$
(\partial c)\left(x_{1}, x_{2}\right)=t c\left(x_{1}\right)+(1-t) c\left(x_{2}\right)-c\left(x_{1} * x_{2}\right),
$$

and in the group case (but with the trivial group action giving a central extension), we put $t=s=1$ and get

$$
(\partial c)\left(x_{1}, x_{2}\right)=c\left(x_{2}\right)-c\left(x_{1} * x_{2}\right)+c\left(x_{1}\right) .
$$


Now we will give a general definition of entropic homology which extends the above for $s=t=0$. For a given entropic magma $(X ; *)$ and $n \geq 0$, let $C_{n}(X)$ be the free abelian group generated by $2^{n}$-tuples $\left(x_{1}, x_{2}, \ldots, x_{2^{n}}\right)$ of elements of $X$; in other words, $C_{n}(X)=\mathbb{Z} X^{2^{n}}=(\mathbb{Z} X)^{\otimes 2^{n}}$. We set $C_{n}(X)=0$ for $n<0$. We are going to define several boundary homomorphisms going from $C_{n}(X)$ to $C_{n-1}(X)$.

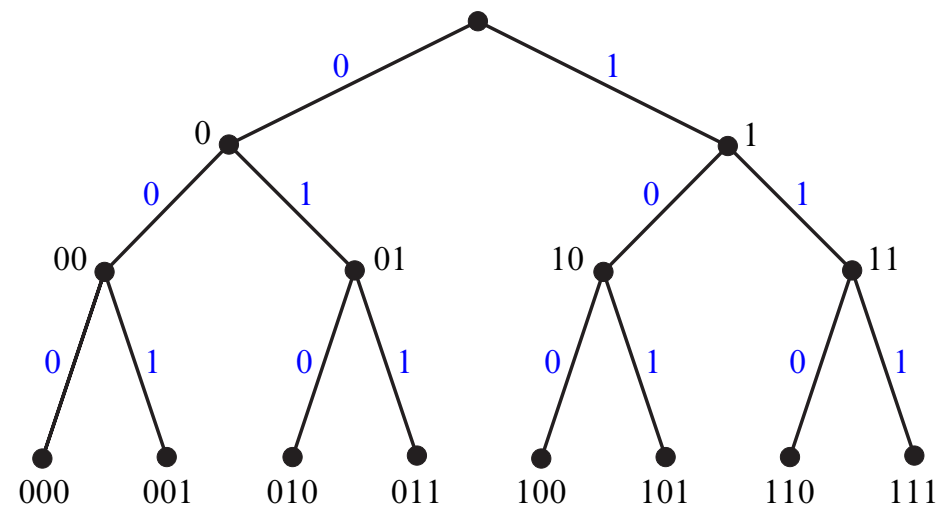

Figure 8: Addresses of nodes in a binary tree

Let $T_{n}$ be the $2^{n}$-tuple of addresses of nodes on the $n^{\text {th }}$ level of the infinite binary tree. For example, $T_{3}=\{000,001,010,011,100,101,110,111\}$ (see Figure 8). We view $T_{n}$ as a $2^{n}$-element set with elements numbered 1 through $2^{n}$. For brevity, we write

$$
w[i]_{1}=k
$$

when the $i^{\text {th }}$ place on the list $w$ has an address containing $k$ ones. Thus, for example, $T_{3}[4]_{1}=2$. Let $S_{2^{n}}^{k}$ denote the set of permutations of the symmetric group $S_{2^{n}}$ that permute only addresses containing $k 1$ s. For example,

$$
S_{2^{3}}^{1}=\{(),(3,5),(2,3),(2,3,5),(2,5,3),(2,5)\},
$$

where ( ) is the identity permutation. For a $2^{n}$-tuple $w$ of elements of $X$, let $\sigma w$ be the result of applying the permutation $\sigma$ to $w$; we extend this linearly to the whole $C_{n}$. We define a homomorphism $\delta_{n}^{k}: \mathbb{Z} X^{2^{n}} \rightarrow \mathbb{Z} X^{2^{n}}$ by

$$
\delta_{n}^{k}(w)=\sum_{\sigma \in S_{2^{n}}^{k}} \operatorname{sgn}(\sigma) \sigma w,
$$

where $\operatorname{sgn}(\sigma)$ denotes the sign of the permutation $\sigma$. Also, for a list $v$ of integers $\left(a_{1}, \ldots, a_{n-1}\right)$, we define $\delta_{n}^{\nu}: \mathbb{Z} X^{2^{n}} \rightarrow \mathbb{Z} X^{2^{n}}$ by

$$
\delta_{n}^{v}(w)=\sum_{i \in\{1, \ldots, n-1\}} a_{i} \delta_{n}^{i}(w) .
$$


For example,

$$
\begin{aligned}
\delta_{3}^{(2,1)}=3()+2(2,3,5)+2(2,5,3)- & 2(3,5)-2(2,3)-2(2,5) \\
& +(4,6,7)+(4,7,6)-(6,7)-(4,6)-(4,7) .
\end{aligned}
$$

We will also need a homomorphism $\mu_{n}: \mathbb{Z} X^{2^{n}} \rightarrow \mathbb{Z} X^{2^{n-1}}$ defined on $2^{n}$-tuples by

$$
\mu_{n}\left(x_{1}, x_{2}, x_{3}, x_{4}, \ldots, x_{2^{n}-1}, x_{2^{n}}\right)=\left(x_{1} * x_{2}, x_{3} * x_{4}, \ldots, x_{2^{n}-1} * x_{2^{n}}\right) .
$$

We will show that the maps $\partial_{n}^{k}=\mu_{n} \delta_{n}^{k}$, with $k \in\{1, \ldots, n-1\}$, play the role of basic differentials.

Theorem 5.1 For $k \in\{1, \ldots, n-1\}, l \in\{1, \ldots, n-2\}$ and $n>2$, we have $\partial_{n-1}^{l} \partial_{n}^{k}=0$.

Proof First we prove that $\mu_{n-1}() \mu_{n} \delta_{n}^{k}=\mu_{n-1} \partial_{n}^{k}=0$, for any $n>1$ and $k \in$ $\{1, \ldots, n-1\}$. It is a useful fact when defining the first homology group. One of the properties of the binary tree is that if the addresses in $T_{n}$, with $n>1$, are divided into 4-tuples, then the middle two addresses in each 4-tuple have the same number of ones. Moreover, for each $k \in\{1, \ldots, n-1\}, n>1$, there is at least one 4-tuple such that the middle two addresses have exactly $k 1 \mathrm{~s}$. Let $i, i+1$ be the positions of such addresses, so $T_{n}[i]_{1}=T_{n}[i+1]_{1}=k$. Then $(i, i+1) \in S_{2^{n}}^{k}$. The map $\sigma \mapsto(i, i+1) \sigma$ gives a bijection between even and odd permutations of $S_{2^{n}}^{k}$. The entropic condition (used after applying $\mu_{n-1} \mu_{n}$ ) ensures that for any $2^{n}$-tuple $w$, we have $\mu_{n-1} \mu_{n}(i, i+1) \sigma w=\mu_{n-1} \mu_{n} \sigma w$ for any $\sigma \in S_{2^{n}}^{k}$. Thus,

that is, $\mu_{n-1}() \mu_{n} \delta_{n}^{k} w=0$.

$$
\mu_{n-1} \mu_{n} \sum_{\sigma \in S_{2^{n}}^{k}} \operatorname{sgn}(\sigma) \sigma w=0,
$$

Now we will show that for $k \in\{1, \ldots, n-1\}, l \in\{1, \ldots, n-2\}, n>2$, and any $\rho \in S_{2^{n-1}}^{l}$, we have $\mu_{n-1} \rho \mu_{n} \delta_{n}^{k}=\mu_{n-1} \rho \partial_{n}^{k}=0$. In the infinite binary tree, every node with an address containing $l 1 \mathrm{~s}$ has two branches leading from it; the left branch leads to a node with an address having $l 1 \mathrm{~s}$, the right branch leads to a node with $l+11 \mathrm{~s}$. The map $\mu_{n}$ corresponds to going up the binary tree, from $T_{n}$ to $T_{n-1}$. It follows that if $x_{i} * x_{i+1}$ is an element in a $2^{n-1}$-tuple $\mu_{n} w$ that has a position with an address containing $l 1 \mathrm{~s}$, then $w[i]_{1}=l$ and $w[i+1]_{1}=l+1$. Thus, any permutation $\rho \in S_{2^{n-1}}^{l}$ determines two permutations $\rho_{1} \in S_{2^{n}}^{l}$ and $\rho_{2} \in S_{2^{n}}^{l+1}$ such that $\rho \mu_{n}=\mu_{n} \rho_{1} \rho_{2}$. It now follows that

$$
\mu_{n-1} \rho \mu_{n} \delta_{n}^{k} w=\mu_{n-1} \mu_{n} \rho_{1} \rho_{2} \sum_{\sigma \in S_{2^{n}}^{k}} \operatorname{sgn}(\sigma) \sigma w=\mu_{n-1} \mu_{n} \sum_{\sigma \in S_{2^{n}}^{k}} \operatorname{sgn}(\sigma) \rho_{1} \rho_{2} \sigma w=0 .
$$


The last equality is true because if both $\rho_{1}$ and $\rho_{2}$ do not permute addresses with $k$ 1s, then $\rho_{1} \rho_{2} \sigma w=\sigma \rho_{1} \rho_{2} w$, and we can use the first part of the proof applied to a $2^{n}$-tuple $\rho_{1} \rho_{2} w$. If one of them, say $\rho_{1}$, permutes the addresses with $k 1 \mathrm{~s}$, then

$$
\sum_{\sigma \in S_{2^{n}}^{k}} \operatorname{sgn}(\sigma) \rho_{1} \rho_{2} \sigma w=\operatorname{sgn}\left(\rho_{1}\right) \sum_{\sigma \in S_{2^{n}}^{k}} \operatorname{sgn}\left(\rho_{1} \sigma\right) \rho_{1} \sigma \rho_{2} w,
$$

and again the equality holds because we take the sum over all permutations from $S_{2^{n}}^{k}$. Now, since $\delta_{n-1}^{l}$ in $\partial_{n-1}^{l}$ is just a sum of signed permutations $\rho \in S_{2^{n-1}}^{l}$, it follows that $\partial_{n-1}^{l} \partial_{n}^{k}=0$.

For a map $\delta_{n}^{v}$ as defined before, let $\partial_{n}^{v}=\mu_{n} \delta_{n}^{v}$.

Corollary 5.2 For any $\alpha \in \mathbb{Z}^{n-1}$ and $\beta \in \mathbb{Z}^{n-2}$, we have $\partial_{n-1}^{\beta} \partial_{n}^{\alpha}=0$.

By selecting a sequence of differentials $d_{n}=\partial_{n}^{v}$ for $n>1, d_{1}=\mu_{1}$ and $d_{i}=$ 0 for $i \leq 0$, we obtain a chain complex $\left\{C_{n}(X), d_{n}\right\}$. The (co)homology of this chain complex is called the entropic (co)homology. For an abelian group $G$, define the chain complex $C_{*}(X ; G)=C_{*} \otimes G$, with $d^{\prime}=d \otimes i d$. The groups of cycles and boundaries are denoted respectively by $\operatorname{Ker}\left(d_{n}\right)=Z_{n}(X ; G) \subset C_{n}(X ; G)$ and $\operatorname{Im}\left(d_{n+1}\right)=B_{n}(X ; G) \subset C_{n}(X ; G)$. The $n^{\text {th }}$ entropic homology group of the entropic magma $(X ; *)$ with coefficient group $G$ is defined as

$$
H_{n}(X ; G)=H_{n}\left(C_{*}(X ; G)\right)=Z_{n}(X ; G) / B_{n}(X ; G) .
$$

Lemma 5.3 Let $w=\left(x_{1}, \ldots, x_{2^{n}}\right) \in C_{n}$ satisfy the following condition: there are $i$ and $j, i \neq j$, such that $w[i]_{1}=k, w[j]_{1}=k$, for some $k \in\{1, \ldots, n-1\}$, and $x_{i}=x_{j}$. Then $\delta_{n}^{k}(w)=0$ (and thus also $\partial_{n}^{k}(w)=0$ ).

Proof If the above condition holds, then for any $\sigma \in S_{2^{n}}^{k}$ we have $\sigma(i, j) w=\sigma w$. It follows that

$$
\delta_{n}^{k}(w)=\sum_{\sigma \in S_{2^{n}}^{k}} \operatorname{sgn}(\sigma) \sigma w=\sum_{\substack{\sigma \in S_{2^{n}}^{k} \\ \operatorname{sgn}(\sigma)=1}} \sigma w-\sum_{\substack{\sigma \in S_{2^{n}}^{k} \\ \operatorname{sgn}(\sigma)=-1}} \sigma w=\sum_{\substack{\sigma \in S_{2^{n}}^{k} \\ \operatorname{sgn}(\sigma)=1}} \sigma w-\sum_{\substack{\sigma \in S_{2^{n}}^{k} \\ \operatorname{sgn}(\sigma)=-1}} \sigma(i, j) w=0,
$$

because the second sum is now over all even permutations $\sigma(i, j) \in S_{2^{n}}^{k}$.

Corollary 5.4 Let $v=\left(a_{1}, \ldots, a_{n-1}\right) \in \mathbb{Z}^{n-1}$ and $w=\left(x_{1}, \ldots, x_{2^{n}}\right) \in C_{n}$. Then $\partial_{n}^{v}(w)=0$ if $w$ satisfies the following condition: For every $i \in\{1, \ldots, n-1\}$ with $a_{i} \neq 0$, there are $x_{r_{i}}$ and $x_{s_{i}}, r_{i} \neq s_{i}$, such that

$$
w\left[r_{i}\right]_{1}=i, \quad w\left[s_{i}\right]_{1}=i \quad \text { and } \quad x_{r_{i}}=x_{s_{i}} .
$$


For example, any tuple $\left(x_{1}, a, x_{3}, b, a, x_{6}, b, x_{8}\right)$ is a cycle in $Z_{3}(X ; \mathbb{Z})$.

It is useful to have a homomorphism $\xi_{n}^{k}: \mathbb{Z} X^{2^{n}} \rightarrow \mathbb{Z} X^{2^{n}}$, with $k \in\{1, \ldots, n-1\}$, defined on $2^{n}$-tuples $w$ by

$$
\xi_{n}^{k} w=(s, s+1) w+w,
$$

where $s$ is the first place in $w$ such that $s=4 i+2$, for some $i$, and $w[s]_{1}=w[s+1]_{1}=$ $k$. In other words, for $w$ divided into 4-tuples, $\xi_{n}^{k}$ finds the first 4-tuple in which the two middle places have $k$ ones in their addresses, permutes the corresponding elements, and adds $w$, so that $\xi_{n}^{k} w$ is a fixed element of the permutation $(s, s+1)$.

Lemma 5.5 For any $w=\left(x_{1}, \ldots, x_{2^{n}}\right) \in C_{n}$, we have $\delta_{n}^{k} \xi_{n}^{k} w=0$ (and $\partial_{n}^{k} \xi_{n}^{k} w=0$ ). Further, for any $v=\left(a_{1}, \ldots, a_{n-1}\right) \in \mathbb{Z}^{n-1}, \partial_{n}^{v} \xi_{n}^{n-1} \cdots \xi_{n}^{2} \xi_{n}^{1} w=0$; here $\xi_{n}^{i}$ can be omitted if $a_{i}=0$.

Proof $\delta_{n}^{k} \xi_{n}^{k} w=0$ because for any $\sigma \in S_{2^{n}}^{k}, \sigma(s, s+1) \xi_{n}^{k} w=\sigma \xi_{n}^{k} w$, and $\sigma \mapsto$ $\sigma(s, s+1)$ is a bijection between even and odd permutations. This holds for any $k \in\{1, \ldots, n-1\}$ if we take $\xi_{n}^{n-1} \ldots \xi_{n}^{2} \xi_{n}^{1} w$ instead of $\xi_{n}^{k} w$, thus, the second statement is true.

It follows that every chain from $C_{n}(X)$ can by made into a cycle by adjusting it with respect to every map $\delta_{n}^{k}$ appearing in the differential $\partial_{n}^{v}$. In particular, a sequence of elements of an entropic magma assigned to the last level of the resolving tree of a link (as described in Section 3) determines a cycle in the entropic homology of the magma. The topological meaning of this correspondence will be investigated in the future work, for now we consider an example.

Example 5.6 Let $\mathcal{A}=\left(A, *, a_{1}, a_{2}, \ldots\right)$ be an entropic magma with a sequence of elements satisfying the conditions of Section 3. The last level of the resolving tree of the framed link 8 , with the left crossing being resolved first, is:
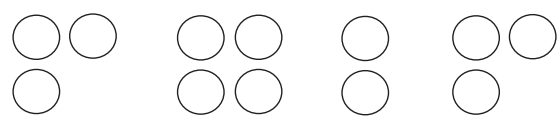

It corresponds to a list $w=\left(a_{3}, a_{4}, a_{2}, a_{3}\right) \in C_{2}(A)$. In general, this list is not a cycle, but $\xi_{2}^{1} w=\left(a_{3}, a_{2}, a_{4}, a_{3}\right)+\left(a_{3}, a_{4}, a_{2}, a_{3}\right)$ is:

$$
\begin{aligned}
\partial_{2}^{(1)} \xi_{2}^{1} w=\mu_{2} \delta_{2}^{1} \xi_{2}^{1} w & =\mu_{2}((()-(2,3))((2,3) w+w)) \\
& =\mu_{2}(()(2,3) w+() w-(2,3)(2,3) w-(2,3) w) \\
& =\mu_{2}(0)=0 .
\end{aligned}
$$


Let $\mathcal{A}$ be the entropic magma

\begin{tabular}{l|llll}
$*$ & 1 & 2 & 3 & 4 \\
\hline 1 & 2 & 1 & 3 & 4 \\
2 & 1 & 4 & 3 & 2 \\
3 & 3 & 3 & 3 & 3 \\
4 & 4 & 2 & 3 & 1
\end{tabular}

with the sequence of elements $a_{1}=1, a_{2}=2, a_{3}=4, a_{4}=1, a_{5}=2, a_{6}=4, \ldots$ Then $w=(4,1,2,4)$ and calculations in GAP [4] show that

$$
\xi_{2}^{1} w=(4,1,2,4)+(4,2,1,4)
$$

is one of the free generators of $H_{2}(\mathcal{A})=\operatorname{Ker}\left(\partial_{2}^{(1)}\right) / \operatorname{Im}\left(\partial_{3}^{(1,0)}\right)$.

Now suppose that we are given a finite family $\left\{X_{i}=\left(X, *_{i}\right)\right\}$ of entropic magmas with an underlying set $X$, satisfying the compatibility condition

$$
\left(a *_{i} b\right) *_{j}\left(c *_{i} d\right)=\left(a *_{j} c\right) *_{i}\left(b *_{j} d\right)
$$

for any elements $a, b, c, d \in X$. In this situation we change the notation for the map $\mu_{n}$. Let $\mu_{n}^{*_{i}}: \mathbb{Z} X^{2^{n}} \rightarrow \mathbb{Z} X^{2^{n-1}}$ be defined by

$$
\mu_{n}^{*_{i}}\left(x_{1}, x_{2}, x_{3}, x_{4}, \ldots, x_{2^{n}-1}, x_{2^{n}}\right)=\left(x_{1} *_{i} x_{2}, x_{3} *_{i} x_{4}, \ldots, x_{2^{n}-1} *_{i} x_{2^{n}}\right) .
$$

For $X_{1}, \ldots, X_{r}$ as above, let $\tau=\left(\tau_{1}, \ldots, \tau_{r}\right) \in\{+,-\}^{r}$ (basically, we are fixing the sign of each operation $\left.*_{i}\right)$. Then we define

$$
\mu_{n}^{\tau}=\sum_{i \in\{1, \ldots, r\}} \tau_{i} \mu_{n}^{*_{i}}
$$

Also, let $\partial_{n}^{k, *_{i}}=\mu_{n}^{*_{i}} \delta_{n}^{k}$ and $\partial_{n}^{k, \tau}=\mu_{n}^{\tau} \delta_{n}^{k}$ for $k \in\{1, \ldots, n-1\}, \partial_{n}^{v, *_{i}}=\mu_{n}^{*_{i}} \delta_{n}^{v}$ and $\partial_{n}^{\nu, \tau}=\mu_{n}^{\tau} \delta_{n}^{\nu}$ for $\nu \in \mathbb{Z}^{n-1}$. The map $\partial_{n}^{\nu, \tau}$ is obtained by adding (or subtracting, depending on $\tau$ ) the considered earlier differentials $\partial_{n}^{v}$ for each operation $*_{i}, i \in$ $\{1, \ldots, r\}$. A simple check gives the following lemma.

\section{Lemma 5.7}

$$
\mu_{1}^{\tau} \partial_{2}^{\nu, \tau}=\mu_{1}^{\tau} \mu_{2}^{\tau} \delta_{2}^{\nu}=0
$$

This leads to the "untwisted" (with $s=t=1$ ) version of the entropic cocycle condition that we considered at the beginning of this section:

$$
\begin{aligned}
f\left(x_{1}, x_{2}\right)-f\left(x_{1}, x_{3}\right)+f\left(x_{3}, x_{4}\right) & -f\left(x_{2}, x_{4}\right) \\
& +f\left(x_{1} * x_{2}, x_{3} * x_{4}\right)-f\left(x_{1} * x_{3}, x_{2} * x_{4}\right)=0 .
\end{aligned}
$$


Here we used three entropic operations: $*_{1}=*$, and the two trivial ones, $a *_{L} b=a$, $a *_{R} b=b$, for all $a$ and $b$, with $\tau=\{+,-,-\}$.

In general, $\mu_{n-1}^{\tau} \partial_{n}^{\nu, \tau} \neq 0$. We can however make a change that leads to a setting in which this kind of condition holds. Let us define a homomorphism $\zeta_{n}: \mathbb{Z} X^{2^{n}} \rightarrow \mathbb{Z} X^{2^{n}}$ defined on $2^{n}$-tuples by

$$
\begin{aligned}
& \zeta_{n}\left(x_{1}, x_{2}, x_{3}, x_{4}, \ldots, x_{4 i}, x_{4 i+1}, x_{4 i+2}, x_{4 i+3}, \ldots, x_{2^{n}-3}, x_{2^{n}-2}, x_{2^{n}-1}, x_{2^{n}}\right) \\
& \quad=\left(x_{1}, x_{3}, x_{2}, x_{4}, \ldots, x_{4 i}, x_{4 i+2}, x_{4 i+1}, x_{4 i+3}, \ldots, x_{2^{n}-3}, x_{2^{n}-1}, x_{2^{n}-2}, x_{2^{n}}\right) .
\end{aligned}
$$

The involution $\zeta_{n}$ appears naturally when we have a family of compatible entropic magmas, namely

$$
\mu_{n-1}^{*_{i}} \mu_{n}^{*_{j}} w=\mu_{n-1}^{*_{j}} \mu_{n}^{*_{i}} \zeta_{n} w .
$$

Now we change the maps $\delta_{n}^{k}(w)$. Let

$$
\hat{\delta}_{n}^{k} w=\sum_{\sigma \in S_{2^{n}}^{k}} \operatorname{sgn}(\sigma)\left(\sigma w-\zeta_{n} \sigma w\right) .
$$

We note that $\zeta_{n} \hat{\delta}_{n}^{k} w=-\hat{\delta}_{n}^{k} w$.

Because of the above change in the definition of $\delta_{n}^{k}(w)$, we slightly change the notation for the remaining maps:

For $k \in\{1, \ldots, n-1\}$ :

$$
\hat{\partial}_{n}^{k, *_{i}}=\mu_{n}^{*_{i}} \hat{\delta}_{n}^{k} \quad \text { and } \quad \hat{\partial}_{n}^{k, \tau}=\mu_{n}^{\tau} \hat{\delta}_{n}^{k}
$$

For $v=\left(a_{1}, \ldots, a_{n-1}\right) \in \mathbb{Z}^{n-1}$ :

$$
\begin{aligned}
& \hat{\delta}_{n}^{v}=\sum_{i \in\{1, \ldots, n-1\}} a_{i} \hat{\delta}_{n}^{i}, \\
& \hat{\partial}_{n}^{\nu, *_{i}}=\mu_{n}^{*_{i}} \widehat{\delta}_{n}^{v} \text { and } \hat{\partial}_{n}^{\nu, \tau}=\mu_{n}^{\tau} \widehat{\delta}_{n}^{v} .
\end{aligned}
$$

Theorem 5.8 For any $v=\left(a_{1}, \ldots, a_{n-1}\right) \in \mathbb{Z}^{n-1}$ and $\tau=\left(\tau_{1}, \ldots, \tau_{r}\right) \in\{+,-\}^{r}$, we have

$$
\mu_{n-1}^{\tau} \widehat{\partial}_{n}^{\nu, \tau}=\mu_{n-1}^{\tau} \mu_{n}^{\tau} \widehat{\delta}_{n}^{\nu}=0
$$

Proof For any $k \in\{1, \ldots, n-1\}$,

$$
\mu_{n-1}^{\tau} \hat{\partial}_{n}^{k, \tau}=\mu_{n-1}^{\tau} \mu_{n}^{\tau} \hat{\delta}_{n}^{k}=\sum_{i \in\{1, \ldots, r\}} \tau_{i} \mu_{n-1}^{*_{i}} \sum_{i \in\{1, \ldots, r\}} \tau_{i} \mu_{n}^{*_{i}} \hat{\delta}_{n}^{k}
$$

We have

$$
\mu_{n-1}^{*_{i}} \mu_{n}^{*_{i}} \hat{\delta}_{n}^{k}=\mu_{n-1}^{*_{i}} \mu_{n}^{*_{i}} \zeta_{n} \hat{\delta}_{n}^{k}=-\mu_{n-1}^{*_{i}} \mu_{n}^{*_{i}} \hat{\delta}_{n}^{k} .
$$

Thus, $\mu_{n-1}^{*_{i}} \mu_{n}^{*_{i}} \widehat{\delta}_{n}^{k}=0$. Similarly,

$$
\mu_{n-1}^{*_{i}} \mu_{n}^{*_{j}} \hat{\delta}_{n}^{k}=\mu_{n-1}^{*_{j}} \mu_{n}^{*_{i}} \zeta_{n} \hat{\delta}_{n}^{k}=-\mu_{n-1}^{*_{j}} \mu_{n}^{*_{i}} \hat{\delta}_{n}^{k} .
$$


Thus, $\mu_{n-1}^{\tau} \widehat{\partial}_{n}^{k, \tau}=0$. Also, $\mu_{n-1}^{\tau} \widehat{\partial}_{n}^{\nu, \tau}=\sum_{k \in\{1, \ldots, n-1\}} a_{k} \mu_{n-1}^{\tau} \mu_{n}^{\tau} \hat{\delta}_{n}^{k}$, so the result follows.

Fixing a $\tau=\left(\tau_{1}, \ldots, \tau_{r}\right) \in\{+,-\}^{r}$, and choosing a sequence of homomorphisms $\widehat{\partial}_{n}^{\nu, \tau}$ for $n>1$, allows us to define groups

$$
\hat{H}_{n}(X)=\operatorname{Ker}\left(\mu_{n}^{\tau}\right) / \operatorname{Im}\left(\hat{\partial}_{n+1}^{\nu, \tau}\right)=\operatorname{Ker}\left(\mu_{n}^{\tau}\right) / \operatorname{Im}\left(\mu_{n+1}^{\tau} \hat{\delta}_{n+1}^{v}\right)
$$

for $n>0$, and

$$
\widehat{H}_{0}(X)=C_{0}(X) / \operatorname{Im}\left(\mu_{1}^{\tau}\right) .
$$

The maps involved in this definition are depicted in the following diagram:

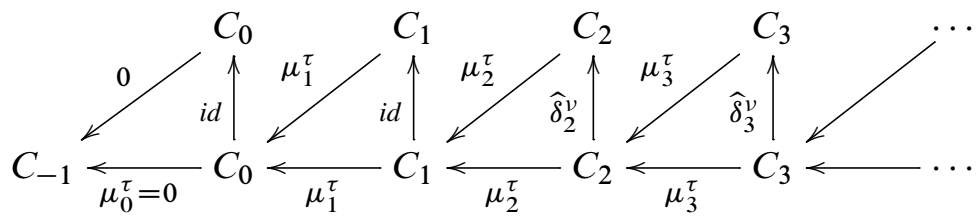

We remark that we could also use $\tau^{\prime}=(-1)^{n} \tau$ instead of $\tau$ in the above definition.

Calculations of $\widehat{H}_{n}$ in GAP show that these groups frequently have torsion elements. We were able to calculate some groups $\hat{H}_{1}$ and $\hat{H}_{2}$, and the torsion parts included powers of $\mathbb{Z}_{2}, \mathbb{Z}_{3}$ and $\mathbb{Z}_{4}$; we expect different $\mathbb{Z}_{k}$ s for higher groups $\hat{H}_{n}$.

Acknowledgements J H Przytycki was partially supported by the NSA-AMS 09111 grant and by the GWU REF grant. He thanks the University of Gdańsk for its hospitality during the summer of 2013.

\section{References}

[1] H Cartan, S Eilenberg, Homological algebra, Princeton University Press (1956) MR0077480

[2] J S Carter, M Elhamdadi, M Saito, Twisted quandle homology theory and cocycle knot invariants, Algebr. Geom. Topol. 2 (2002) 95-135 MR1885217

[3] I M H Etherington, Non-associative arithmetics, Proc. Roy. Soc. Edinburgh. Sect. A. 62 (1949) 442-453 MR0029898

[4] GAP Group, GAP - Groups, algorithms, programming (2013) Available at http:// www.gap-system.org

[5] J B Listing, Vorstudien zur Topologie, Vandenhoeck und Ruprecht, Göttingen (1847)

[6] D C Murdoch, Quasi-groups which satisfy certain generalized associative laws, Amer. J. Math. 61 (1939) 509-522 
[7] J H Przytycki, Survey on recent invariants on classical knot theory arXiv 0810. 4191

[8] J H Przytycki, From Goeritz matrices to quasi-alternating links, from: "The mathematics of knots", (M Banagl, D Vogel, editors), Contrib. Math. Comput. Sci. 1, Springer, Heidelberg (2011) 257-316 MR2777853

[9] J H Przytycki, KNOTS: From combinatorics of knot diagrams to the combinatorial topo logy based on knots, Cambridge Univ. Press (2014) to appear

[10] J H Przytycki, P Traczyk, Conway algebras and skein equivalence of links, Proc. Amer. Math. Soc. 100 (1987) 744-748 MR894448

[11] J H Przytycki, P Traczyk, Invariants of links of Conway type, Kobe J. Math. 4 (1988) 115-139 MR945888

[12] A K Sushkevich, The theory of generalized groups, Gos. Nauchn. Tekhn. Izd., KharkovKiev (1937)

[13] K Toyoda, On affine geometry of abelian groups, Proc. Imp. Acad. Tokyo 16 (1940) 161-164 MR0002145

[14] K Toyoda, On axioms of mean transformations and automorphic transformations of abelian groups, Tôhoku Math. J. 46 (1940) 239-251 MR0002144

[15] K Toyoda, On linear functions of abelian groups, Proc. Imp. Acad. Tokyo 16 (1940) 524-528 MR0004038

[16] K Toyoda, On axioms of linear functions, Proc. Imp. Acad. Tokyo 17 (1941) 221-227 MR0014105

Department of Mathematics, University of Louisiana at Lafayette

217 Maxim D Doucet Hall, 1403 Johnston Street, Lafayette, LA 70504-1010, USA

Department of Mathematics, The George Washington University

Monroe Hall, Room 240, 2115 G Street NW, Washington, DC 20052, USA

mniebrz@gmail.com, przytyck@gwu.edu

http://home.gwu.edu/ przytyck/

Received: 19 September 2012 